\title{
CITY BRANDING COMO ESTRATEGIA DE MERCADO PARA PROMOCIONAR EL TURISMO EN LA CIUDAD DE IPIALES
}

\section{CITY BRANDING AS A MARKET STRATEGY TO PROMOTE TOURISM IN THE CITY OF IPIALES}

\section{CIDADE BRANDING COMO ESTRATÉGIA DE MERCADO PARA PROMOVER O TURISMO NA CIDADE DE IPIALES}

\section{ARTEAGA FLÓREZ_Andrea Lorena, PIANDA ESTRADA_Edith Marcela, SANDOVAL MONTENEGRO_Ximena Alejandra}

Magister en Administración de Negocios, Escuela Superior de Economía y Administración de Empresas ESEADE, Docente Investigadora de la Maestría en Administración. Universidad Mariana, Jefe GrupLAC grupo de investigación CONTAR. Email: andrealafz@gmail.com, Colombia.

Candidata a Magister de la Maestría en Mercadeo. Administradora de Negocios Internacionales Universidad Mariana. Email: marcelapianda@gmail.com, Colombia.

Candidata a Magister de la Maestría en Mercadeo. Administradora de Negocios. Universidad San Buenaventura. Email: xime_aleja1808@hotmail.com, Colombia.

DOI: http://dx.doi.org/10.22267/rtend.192001.106 


\title{
RESUMEN
}

El presente artículo tiene como propósito la creación de una marca ciudad (City Branding) para la ciudad de Ipiales - Nariño, para el año 2019, como estrategia de mercado para promocionar e impulsar el turismo en la ciudad de Ipiales, es así que, este escrito es el resultado de una investigación cuantitativa, pretendiendo dar una explicación de una realidad social con un tipo de estudio descriptivo y un método deductivo, a través de herramientas de recolección de información documental y encuestas a residentes y visitantes de la ciudad en mención.

En primer lugar se identificaron las potencialidades que tiene Ipiales en cuanto a sitios turísticos, en segundo lugar se evaluó la percepción de residentes y visitantes sobre la ciudad, además de identificar atributos importantes de la misma y finalmente, atendiendo a la necesidad de estimular la imagen e identidad de la cuidad de Ipiales, como base para el reconocimiento urbano y posicionamiento nacional, se propuso la creación de una marca que otorgue diferenciación y notoriedad para la ciudad, determinando además que, la creación de una marca para la ciudad de Ipiales resulta pertinente por la ubicación geográfica, los lugares turísticos y el componente histórico y cultural, contribuyendo a fortalecer la dinámica urbana y el desarrollo turístico.

Palabras clave: marca, marca territorial, creación de valor, posicionamiento, turismo.

JEL: M3, R1, R11, M37, L8

\begin{abstract}
The purpose of this paper is to create a city brand (City Branding) for the city of Ipiales - Nariño, as a market strategy to promote and promote tourism in the city of Ipiales, so this writing is the result of a quantitative research, trying to give an explanation of a social reality with a type of descriptive study and a deductive method, through tools of collection of documentary information and surveys to residents and visitors of the mentioned city.
\end{abstract}

In the first place, the potentialities that Ipiales has in terms of tourist sites were identified, secondly, the perception of residents and visitors about the city was 
evaluated, as well as identifying important attributes of the city and finally, taking into account the need to stimulate the image and identity of the city of Ipiales, as a basis for urban recognition and national positioning, the creation of a brand that grants differentiation and notoriety for the city was proposed, determining that, the creation of a brand for the city of Ipiales is relevant by the geographical location, the tourist places and the historical and cultural component, contributing to strengthen the urban dynamics and the tourist development.

Keywords: brand, territorial brand, value creation, positioning, tourism. JEL: M3, R1, R11, M37, L8

\section{RESUMO}

O objetivo deste artigo é criar uma marca de cidade (City Branding) para a cidade de Ipiales - Nariño, como uma estratégia de mercado para promover e promover o turismo na cidade de Ipiales, por isso esta escrita é o resultado de uma pesquisa quantitativa, tentando dar uma explicação de uma realidade social com um tipo de estudo descritivo e um método dedutivo, através de ferramentas de coleta de informações documentais e pesquisas para moradores e visitantes da citada cidade.

Primeiro o potencial que tem Ipiales a locais turísticos, segundo a percepção dos moradores e visitantes sobre a cidade foi avaliada, bem como a identificação de atributos importantes dos mesmos e, finalmente, com a necessidade de estimular a imagem identificada e identidade da cidade de Ipiales, como base para o reconhecimento nacional urbana e posicionamento, a criação de uma marca que dá a diferenciação e notoriedade para a cidade foi proposto, também determina que a criação de uma marca para a cidade de Ipiales é relevante pela localização geográfica, os locais turísticos e o componente histórico e cultural, contribuindo para fortalecer a dinâmica urbana e o desenvolvimento turístico.

Palavras-Chave: marca, marca territorial, criação de valor, posicionamiento, turismo.

JEL: M3, R1, R11, M37, L8 


\section{INTRODUCCIÓN}

Hoy en día las organizaciones, indiferente de su tamaño, localización y sector, invierten en la construcción de su marca, su sostenimiento, y por supuesto en el mantenimiento de su buen nombre, con el fin de posicionarse en el mercado, y por consiguiente avanzar en términos de auto sostenibilidad. De igual manera, las ciudades no son la excepción en esta nueva dinámica, a través de la cual se aporta un significado cultural a la ciudad haciéndola más atractiva y generando oportunidades de negocio.

En efecto, los territorios urbanos también son objeto de actividades y estrategias de marketing; tal como lo afirma Muñiz (2010), las instituciones territoriales como ciudades, regiones, naciones y países del mundo, están adoptando de forma creciente enfoques de gestión estratégica y marketing, con ciertos paralelismos con la gestión empresarial.

En esa misma dirección, vale destacar que actualmente las ciudades, en la medida en que crecen demográficamente, son vistas como polos de desarrollo, que aglutinan innumerables oportunidades y alternativas económicas y sociales, cuyos resultados, transforman no solo a su comunidad propiamente dicha, sino también a su entorno. Como afirma Olins (2004), las ciudades ahora buscan atraer nuevas inversiones, captar nuevas empresas y atraer al turismo lo que en un entorno de máxima competencia se traduce en múltiples posibilidades para el consumidor.

Por otra parte, en el Departamento de Nariño, las actividades turísticas han empezado a tomar una dinámica activa y favorable para el desarrollo económico de la región, siendo el turismo una actividad con gran potencial debido a la posición geoestratégica que le permite su promoción hacia el país del Ecuador, además de contar con atractivos naturales como volcanes, reservas naturales, lagunas, paisajes, convirtiéndose en una ventaja comparativa que permite incursionar dentro de una oferta turística. Sin embargo, el potencial del Departamento de Nariño ha sido poco aprovechado y no se ha constituido como factor diferencial de oferta turística nacional; lo mismo sucede con la ciudad de Ipiales razón por lo cual no hay un incentivo dentro de la rama del turismo que promueva una estrategia de desarrollo significativa para el progreso de la ciudad. 
Es así que, Ipiales al contar con diversos recursos naturales, aunado a una ubicación privilegiada y estratégica desde el punto de vista comercial, cultural y religioso no ha podido desarrollar proyectos que potencialicen sus atractivos turísticos ni tampoco la oferta de servicios para generar valor agregado, situación que incide de manera negativa en torno a la realización de convocatorias de inversión turística nacional y extranjera. Por lo anterior, se propone la creación de la marca ciudad de Ipiales, que permita identificar los valores comunes que puedan existir y potencializarlos de manera que se proyecten y marquen una diferenciación respecto a otros centros urbanos; todo esto representado en un elemento visual que conecte emociones.

En este orden de ideas, para el proceso de creación de la marca ciudad, el artículo se estructura en primer lugar, con la revisión de información documental sobre marca y marca ciudad. Seguido a esto se reconocen las potencialidades que tiene Ipiales en cuanto a lugares de destino e identificación del componente turístico de la ciudad, así como también la necesidad y determinación colectiva de desarrollo turístico que permita crear estrategias de City Branding con las cuales se provean resultados que traigan consigo beneficios y desarrollo económico para la ciudad de Ipiales. Además se pudo obtener percepciones, sugerencias, atributos, comentarios y estadísticas de los residentes y visitantes de la ciudad, mediante la aplicación de encuestas, útiles para la creación de la marca ciudad. Finalmente se propone la creación de una marca que otorgue diferenciación y notoriedad para la ciudad de Ipiales.

\section{METODOLOGÍA}

El artículo es el resultado de una investigación con enfoque cuantitativo y el método de estudio deductivo. El tipo de estudio en función de sus objetivos de investigación es descriptivo. De este modo, se propone crear una marca para la ciudad de Ipiales, para contribuir a que la ciudad potencialice el turismo, generando oportunidades de desarrollo y crecimiento.

En primer lugar, se tomó información secundaria, se realizó una revisión documental con relación a conceptos y teorías sobre marketing, marca, Branding, City Branding; además de caracterizar la actividad Turística de la ciudad de Ipiales. 
En segundo lugar, se recurrió a información primaria, se tomó la población de residentes y visitantes de la ciudad de Ipiales; en este sentido, para determinar la muestra de los residentes de la ciudad, se tomó el total de hogares existentes en la ciudad y se calculó una muestra representativa con el 95\% de confianza para un total de 377 familias con un muestreo probabilístico al azar, de igual manera, se realizó un muestreo no probabilístico por conveniencia a 100 personas que visitaron la ciudad de Ipiales durante más de tres días. Se utilizó la encuesta como una técnica estructurada para recolectar información directamente de los integrantes de una muestra a partir de aplicación de un cuestionario previamente validado, se realizó el trabajo de campo y posterior a ello se recopiló y analizó la información con el objetivo de conocer la percepción de residentes y visitantes de la ciudad de Ipiales sobre valores culturales, humanos, religiosos, elementos simbólicos, gastronómicos y paisajísticos de la ciudad de Ipiales, aspectos claves para la construcción de la marca ciudad.

\section{REVISIÓN DE LA LITERATURA}

Marketing. El termino marketing se empezó a utilizar en Estados Unidos a principios del siglo XX. Según la American Marketing Association define marketing como "un proceso de planificación y ejecución de la concepción, la fijación del precio, promoción y distribución de ideas, bienes y servicios para crear intercambios que satisfagan los objetivos de los individuos y de las organizaciones" (Rovetto, 2007, p. 123)

Seguido a la definición de marketing, es necesario abordar el termino estrategia de marketing, entendido para Rovetto (2007), que la estrategia de marketing debe plantearse en un plan de marketing el cual lo define como:

\footnotetext{
"un documento escrito en el que de una forma sistemática y estructurada y previos los correspondientes análisis y estudios, se definen los objetivos a conseguir en un periodo determinado así como también se detallan los programas y medios de acción que son precisos para alcanzar los objetivos en el plazo previsto", (p. 126)
}

Finalmente, y tratando de abordar el marketing de ciudad, se resume que el marketing se ha aplicado específicamente a productos y servicios, enfocado hacia 
la orientación del cliente y la competencia, y también, gracias a los procesos de globalización y a los tratados de libre comercio, las ciudades deben prepararse para convertirse en sitios atractivos a la inversión nacional y extranjera, al mismo tiempo deben contar con aquellos requisitos que ellos exigen; lo que conlleva a percibir la ciudad como un producto.

Branding y City Branding. La marca se refiere esencialmente a un nombre, termino, símbolo o diseño especial con el que se trata de identificar los bienes y servicios de un vendedor o grupo de vendedores. Una marca registrada es aquella que recibe protección legal debido a que realizo un acuerdo con los establecimientos públicos legalmente establecidos (San Clemente et al, 2009, 129). De acuerdo a los estudios realizados por Bonnet (2015) citado por (Fernández, 2015, Pg.18) define la marca como un término, un nombre, símbolo o diseño o la combinación de ellos, que trata de identificar los bienes o servicios de un grupo de vendedores y diferenciarlos de los competidores. Así mismo, de acuerdo a Kotler (1995), citado por Fernández, la marca la define como "un nombre, un término, señal, símbolo o diseño o a su vez una combinación de ellos con la intensión de identificar los productos y servicios de un vendedor o grupo de vendedores, para diferenciarse de la competencia" (Fernández, 2015, p. 19)

Para Fernández (2015), las marcas influyen en "la vida de los consumidores y son determinantes en la decisión de compra, por lo cual considera indispensable que toda compañía debe realizar una inversión en el Branding de sus productos" (p.40). Es decir, que independientemente de la compañía que exista o empresa, como punto esencial de la vida de toda compañía para subsistir dentro de un mercado competitivo, debe invertir un porcentaje del capital en publicidad, en posicionamiento de la marca, en el manejo simbólico de la empresa y en captar la atención del público.

Los anteriores conceptos permiten configurar lo que es la identidad de la marca, por lo cual la identidad de la marca es un conjunto de atributos vinculados al nombre y símbolo de la marca que incorporan el valor suministrada por un producto o servicio. Así "el concepto de identidad sirve de base para explicar la gran mayoría de conceptos que a nivel corporativo se desarrollan en las empresas tales como la imagen, la reputación o la comunicación corporativa" (Pérez, 2014, p. 100). 
Por otra parte, para Rob Frankl (2005) citado por Bastos al (2015) considera que la teoría del Branding consiste en lograr que una marca sea percibida como la mejor, como la única solución, lo que se traduce en fidelidad del cliente hacia una marca o producto, (p.3). Es decir, que la teoría del Branding busca el posicionamiento de una marca que se adquiere dentro del mercado, logrando trasmitir su personalidad a la mente del consumidor. En cambio para Interbrand, ${ }^{1}$ el Branding consiste en desarrollar y mantener el conjunto de atributos y valores de una marca de tal manera que sean coherentes, apropiados, distintivos, susceptibles de ser protegidos legalmente y atractivos para los consumidores, (p. 5).

Para Kotler y Amstrong, autores del libro fundamentos del marketing, indican que la marca "es el nombre, señal o diseño con el que se identifica y comercializa un producto o bien o servicio de una empresa u organización y que se diferencia del resto de la competencia" (Bastos et al, 2015). En cambio para Sánchez (2015), la marca es "todo signo susceptible de representación gráfica que sirve para distinguir en el mercado los productos o servicios de una empresa de las demás" (p. 158)

Para Urbina y Palacios, citado por Bastos, las marcas tienen un Ciclo de vida, se crean, se introducen, desarrollan, alcanzando de esta manera un nivel de madurez, que llegando un momento dado sufre algún desgaste, el cual inevitablemente la conduce a un punto de decisión, que es la oportunidad o muerte, es ahí donde los directivos de las empresas deben tomar las decisiones para transformarlas y reinventarlas (Bastos et al, 2015).

Leca (2015), concluye que al revisar definiciones que establece el concepto marca, se relacionan con la pertenencia o propiedad, así como con los beneficios y con cómo se construyen, también con el valor y la diferenciación que aportan a las compañías a las que pertenecen, y con la relación que establecen con los consumidores y la sociedad siendo un elemento clave en la construcción de la reputación de las empresas.

\footnotetext{
${ }^{1}$ Es una consultora de marca que se especializa en áreas como estrategia de marca, análisis de marca, valoración de marca, diseño corporativo, gestión de marca digital, diseño de packaging y naming. Interbrand tiene 24 oficinas en 17 países.
} 
Ahora tomando el termino completo de "City Branding", se resume que la palabra proviene del vocablo ingles "Brand" que significa marca, por lo cual etimológicamente, se puede decir que los productos o servicios que se han de ofrecer al mercado se pueden diferenciar de los demás marcándolos con sello especifico que logre que estos sean reconocidos por la sociedad y crean una preferencia por encima de los demás productos y servicios en el mercado (Rovetto, 2007).

Además de lo anterior, "la ciudad debe ser agradable para sus residentes quienes la conciben como un lugar para vivir; así mismo, las empresas buscan un lugar estratégico e interesante para invertir; y los turistas buscan un lugar de interés cultural, de descanso y entretenimiento" (Mejía \& Bolaño, 2014). Es decir, según Abadía (2015), la marca debe representar algo claro y preciso, debe ser de interés para los residentes y visitantes de una región, y lo más importante tiene que ser creíble en el sentido de vincular a un determinado producto de la ciudad, relacionado con una realidad y un proyecto urbano.

Finalmente, para Rincón, (2010) una marca

"tiene que utilizarse como símbolo de su personalidad y debe estar asociada a una serie de activos y recursos urbanos existentes y a unos valores relacionados con el modelo de ciudad y con una significativa capacidad de atracción: humanismo, bienestar, convivencia, sostenibilidad, solidaridad, libertad, tolerancia, cooperación y respeto al medio ambiente" (p. 13).

Por otra parte, según Fernández (2015), uno de los sectores en los que se aplica la estrategia de marca con mayor frecuencia es a partir del turismo, debido a la necesidad de los destinos para diferenciarse de sus competidores (p.25). Por tanto, el turismo además de disminuir las distancias entre países y territorios, se ha convertido durante los últimos tiempos en una fuente de trabajo y obtención de recursos económicos y en motor inclusive de las economías de los países; tal es el caso de países Europeos como Francia, España, Grecia y otros, que gracias a las políticas de turismo nacionales, han permitido el reconociendo exhaustivo de las ciudades y territorios, por lo que sus economías se mueven ciertamente alrededor de este sector.

Según Fierro et al (2011) infieren que "los planteamientos del City Branding, se han convertido en técnicas esenciales para promocionar y posicionar las ciudades en el mundo, por lo cual la construcción de una marca ha permitido 
amplificar las estrategias de marketing y la diferenciación en un mercado global, mediatizados por la información que se encuentra en internet o en los medios virtuales" (p. 28); por lo cual los gobernantes incorporan la noción del City Branding a través del internet con la intención de construir imágenes positivas para persuadir a los inversionistas y turistas potenciales.

Para Merino (2014), el punto de partida para el estudio del Branding de destinos, se sustenta en los conceptos teóricos que aparecen en el tratado Marketing Places, escritos por Philip Kotler, Donald Haider e Irvin Rein, quienes "explican la relación entre el Branding de ciudades y las ciencias económicas, al hacer referencia a las crisis que presentaron Nueva York y California en los Estados Unidos y Rio de Janeiro en Sur América, ciudades que se declararon en banca rota o en crisis" (p. 39)

Así, las crisis económicas y sociales en el mundo permitieron con el tiempo priorizar acerca del Branding, el cual poco a poco fue aplicado en diferentes países tomando la marca de una ciudad como principal destino turístico, de hecho existen diferentes estrategias que se han aplicado alrededor del mundo obteniendo grandes beneficios no solo en términos económicos sino también en mejoramiento de la calidad de vida, establecimiento de propuestas innovadoras en términos de turismo y por consiguiente, mejorando el ambiente general de las ciudades y el posicionamiento de ellas.

Ejemplo de ello es países como Sudáfrica, el cual durante el año 2000 decide cambiar la percepción que se tenía del país, cuya historia había estado ligada a la injusticia social, emprendiendo un proyecto de marca bajo el lema "Sudáfrica, viva en posibilidades" propuesta sobre una idea de nación que propone posibilidades distintas y que inspira a hacer las cosas diferentes, así mismo se destaca el trabajo que ha hecho Brasil la cual durante el año 2004 saca a relucir la marca "Brasil it!" cuyo objetivo fue posicionar la industria y exportar sus productos al mercado norteamericano.

El anterior ejemplo, es una muestra de cómo el City Branding aplicado a los destinos, contribuye a que los países y ciudades logren ventajas económicas, así como también logren la gestación de sus productos dentro de los mercados internacionales, cambiando de esta manera las percepciones a nivel global. 


\section{RESULTADOS}

En primer lugar se caracteriza la actividad turística de la ciudad de Ipiales; en segundo lugar se da a conocer los aspectos más relevantes sobre la percepción que residentes y visitantes tuvieron sobre valores culturales, religiosos, elementos simbólicos, gastronómicos y paisajísticos de la ciudad y en tercer lugar se propone la Marca Ciudad (City Branding) de la Ciudad de Ipiales además de contemplar estrategias para su promoción.

\subsection{Caracterización de la ciudad de Ipiales.}

La ciudad de Ipiales se caracteriza por tener distintivos respecto a otros municipios fronterizos, fundamentalmente por la infraestructura y a la ubicación geográfica, la cual tiene ciertas ventajas competitivas debido al comercio binacional que se da con el vecino país del Ecuador; también se distingue por tener renglones importantes dentro del sector comercial, financiero, de transporte debido a su frontera transnacional con el país vecino el Ecuador, lo cual es un aporte importante no solo para la economía local y regional, sino también para la economía a nivel nacional.

Un renglón importante dentro de la economía de Ipiales es el servicio de hospedaje, alimentación y transporte, el cual cuenta con hoteles representados en diferentes categorías según el presupuesto del cliente; actualmente Ipiales gracias a los proyectos turísticos y a los procesos de desarrollo comercial fronterizo, ha dado lugar a la generación de la puesta en marcha de ciertos proyectos hoteleros situados en varios sitios de la ciudad, destacándose la construcción de hoteles de primera, segunda y tercera clase . Los cuales cuentan con todos los servicios acordes a las necesidades y capacidades de los clientes. (Plan de desarrollo Municipal 2016 - 2019, Alcaldía Municipal de Ipiales, 2016).

Así mismo, Ipiales cuenta con un número importante de asaderos, restaurantes y cafeterías, los cuales ofrecen una gran variedad de platos típicos e internacionales, brindando una atención al cliente y al turista internacional, destacándose la especialidad de platos típicos de la región como es el caso del cuy, la fritanga, sancocho de costilla, sancocho de gallina, papas, queso, carne de cordero, envueltos, etc. (Plan de desarrollo Municipal 2016 - 2019, Alcaldía Municipal de Ipiales, 2016). 
Otra característica dentro del componente turístico, se encuentra lo que comúnmente se conoce como patrimonio material, dentro de los cuales se destacan los patrimonios arquitectónicos especialmente lo que tiene que ver con arquitectura religiosa; dentro de los cuales el más conocido y el que le ha dado un toque especial al turismo dentro del municipio es el Santuario de las Lajas, santuario que se encuentra ubicado en el cañón del rio Guáitara, edificación religiosa que se construyó el primero de enero de 1795 y que se terminó el 21 de abril de 1803, templo de estilo gótico del siglo XIV, construcción que se hizo con base en rocas similares y calicanto adornado por arcos de medio punto que representan el romanticismo europeo (Alcaldía Municipal de Ipiales, 2016).

Dentro de las obras de ingeniería e infraestructura se destaca la comúnmente conocida como "casona" y el puente natural de Rumichaca (antiguo); dicho puente natural que es un puente de piedra ubicado entre Ipiales y Tulcán sobre el rio Guáitara o Carchi, fue construido por el indígena Huayna Capac; el puente se encuentra unido topográficamente por la grandes piedras que le sirven de base a lado y lado del rio Guáitara (Alcaldía Municipal de Ipiales, 2016).

Dentro de las arquitecturas que conforman o hacen parte del patrimonio cultural material inmueble se encuentra la piedra de los monos la cual contiene figuras zoomorfas y antropomorfas y geométricas abstractas representando pastores y/o danzantes y algunas figuras geométricas, representando algunas de las tradiciones culturales de los indígenas antecesores; también se destaca el monumento a la música andina "el ciego" manual de rivera "la india" Juana de Nueces de Quiñones (Alcaldía Municipal de Ipiales, 2016).

Respecto al patrimonio cultural e inmaterial, se destaca la gastronomía y los saberes culinarios, gastronomía que se destaca por tener comidas típicas como es el caso del cuy, la fritada, el sancocho de gallina, sancocho de costilla, la carne de cordero, papas, ají, etc. ubicadas principalmente en la vía panamericana salida a Pasto en el barrio los chilcos y las cruces, en la vía aeropuerto san Luis y en el barrio el charco.

Otros de los aspectos que guardan cierta relación con los aspectos turísticos del municipio, se encuentran las festividades y los eventos culturales, es decir las fiestas religiosas o patronales, dentro de las cuales se destacan las fiestas de la 
virgen de las lajas, la cual se realiza la segunda semana del mes de septiembre en conmemoración a su aparición. Evento religioso que se caracteriza por ser un novenario en el cual se celebran misas y eventos culturales distribuidos en barrios y veredas, después de la misa se queman castillos y participan diferentes grupos musicales.

\subsection{Turismo que ofrece la Ciudad de Ipiales.}

Dentro de los tipos de turismo actual que posee la ciudad de Ipiales se encuentran regularmente las arquitecturas religiosas representadas en templos, de los cuales se destaca principalmente el Santuario de las Lajas o templo basilisco, así mismo se destaca el cementerio parroquial o de las lajas. Dentro de las obras arquitectónicas para el transporte fluvial y marítimo se destaca la terminal de transporte y aeropuerto de San Luis y el puente natural de Rumichaca. De igual manera existe un gran atractivo turístico relacionado con la gastronomía y los saberes culinarios basadas en comidas típicas como el cuy, la fritada, sancocho de gallina y sancocho de costilla.

Dentro de los atractivos turísticos que pueden ser potenciales a mediano o largo plazo para la ciudad de Ipiales se encuentra el cementerio parroquial o de las lajas, el puente natural de Rumichaca, la piedra de los monos, el monumento a la música andina, los resguardos indígenas de Ipiales Yamaral, San Juan y Santa Rosa de Sucumbió; son algunos de los sitios arquitectónicos culturales que pueden ser aprovechados de una manera formal gracias a la implementación de estudios pertinentes que permitan conducir hacia la elaboración de políticas de desarrollo turístico y la implementación de reconstrucción arquitectónica como es el caso del puente natural de Rumichaca el cual al ser un puente natural de piedra, puede ser considerado un alto componente dentro del sector turístico, teniendo en cuenta su historia, su legado, su proceso y fundamentalmente su trascendencia.

Otro sitio arquitectónico que merece mayor atención y reconocimiento como sitio turístico potencial es "la piedra de los monos", pictografía que al obtener figuras amorfas y antropomorfas y geometrías abstractas, pueden ser un contenido arquitectónico con gran potencial, el cual el cual ha sido objeto de estudios e investigaciones por parte de antropólogos, filósofos y arquitectos, convirtiendo este lugar 
en un espacio geográfico, arquitectónico e inmaterial no solo para los turistas sino también para la gente que reside en el municipio, Siendo dicho sitio una ruta más de los sectores que hacen parte de los atractivos turísticos de Ipiales pero con un componente diferencial que representa el patrimonio material e inmaterial de la humanidad junto a la historia de sociedades y pueblos prehispánicos.

\subsection{Percepción de los residentes y visitantes de la ciudad de Ipiales sobre} atributos, elementos simbólicos y turísticos de la ciudad de Ipiales.

Atributos que identifica a la ciudad. Los residentes de la ciudad consideraron que Ipiales se identifica como ciudad visionaria; de igual manera, los visitantes además de ciudad inspirada en la gente. (Ver figura 1), es así que estos atributos son importantes para crear la marca ciudad y promocionarla, teniendo en cuenta que es considerada una ciudad con proyección y desarrollo.

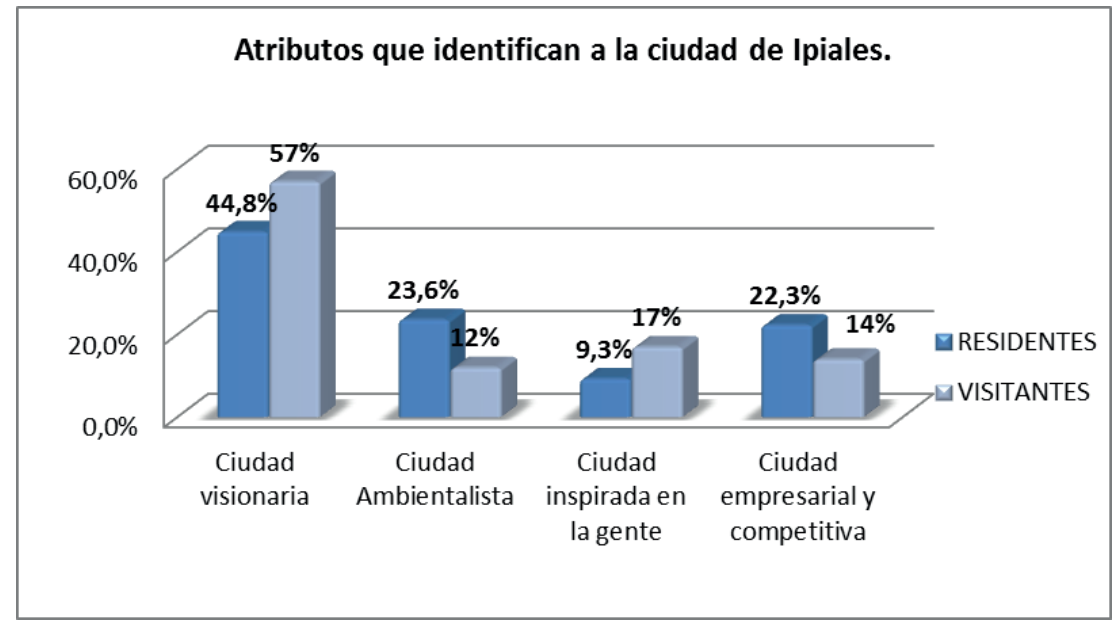

\section{Figura 1}

Atributos de identificación de la ciudad de Ipiales

Fuente: Elaboración propia

Sitios turísticos. En cuanto a los sitios turísticos más visitados por los residentes de Ipiales, se reconoce al santuario de las Lajas, el Barrio el Charco y el Volcán Nevado del Cumbal; la misma preferencia se observa en los visitantes donde afirman que el santuario de las lajas es el lugar más visitado, seguido del Barrio el Charco, así mismo pero en menor proporción existe preferencia sobre el centro recreacional 
Simón Bolívar (Ver figura 2). Información relevante para promocionar los sitios turísticos citados y los restantes, siendo estos un atractivo turístico importante teniendo en cuenta su historia y diferenciación de otros lugares.

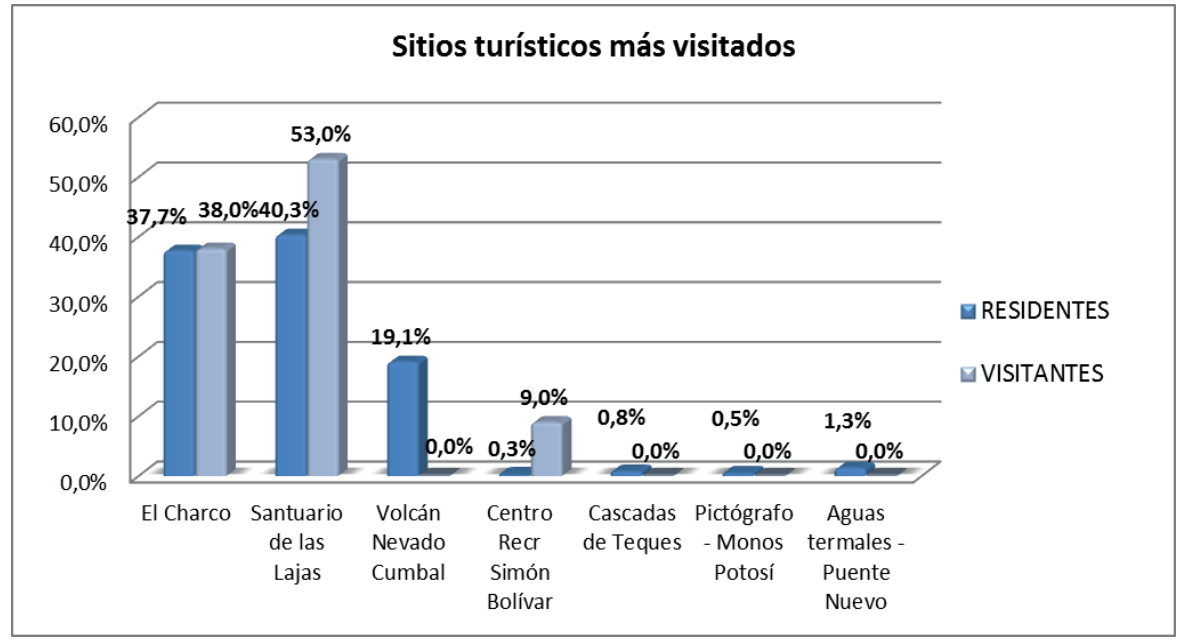

\section{Figura 2}

Sitios turísticos de la ciudad de Ipiales

Fuente: Elaboración propia

Gastronomía. La comida típica más apetecida en su orden es el cuy, la carne de ternera y la fritada, lo cual determina los gustos de los ciudadanos en términos de comidas típicas. Con relación a los platos típicos que consumieron los visitantes, en su mayoría consideraron el cuy, seguido de la carne de ternera y la fritada. (Ver Figura 3). Resultados que determinan la escogencia y predilección de ciertos platos típicos por parte de la población objeto de estudio, además esta información es útil para fomentar y promocionar la comida típica tradicional y deliciosa no mencionada como el helado de paila, Lapingachos, Pan de maíz y Champús.

Además, se preguntó respecto a la gastronomía de la ciudad, los visitantes encuestados respondieron en su mayoría como muy buena con y buena, determinando que uno de los aspectos privilegiados es la aceptación de sus platos típicos. 


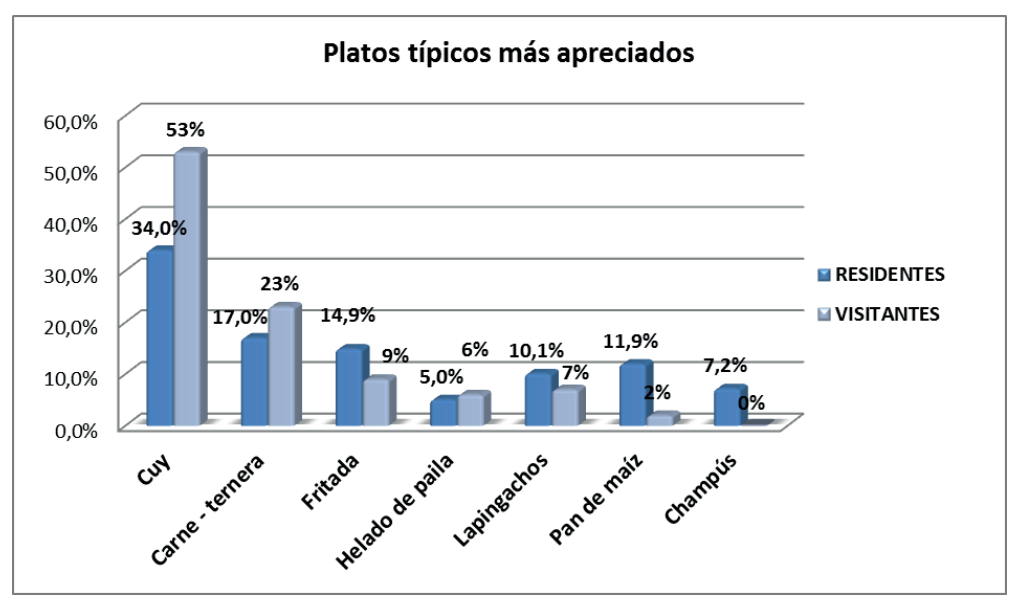

\section{Figura 3}

Gastronomía de la ciudad de Ipiales

Fuente: Elaboración propia

Seguridad, limpieza y orden en la ciudad. Los residentes de Ipiales, consideraron que la ciudad no es segura, de igual manera afirmaron que la ciudad tiene problemas de limpieza y orden. La percepción de inseguridad no es tan notoria en los visitantes, pero igual presente problemas de orden y limpieza. Es importante para crear una marca ciudad que la ciudad brinde seguridad y condiciones agradables, por lo tanto se debe proponer acciones para mejorar esta imagen ahora desfavorable (Ver figura 4 y 5 ).

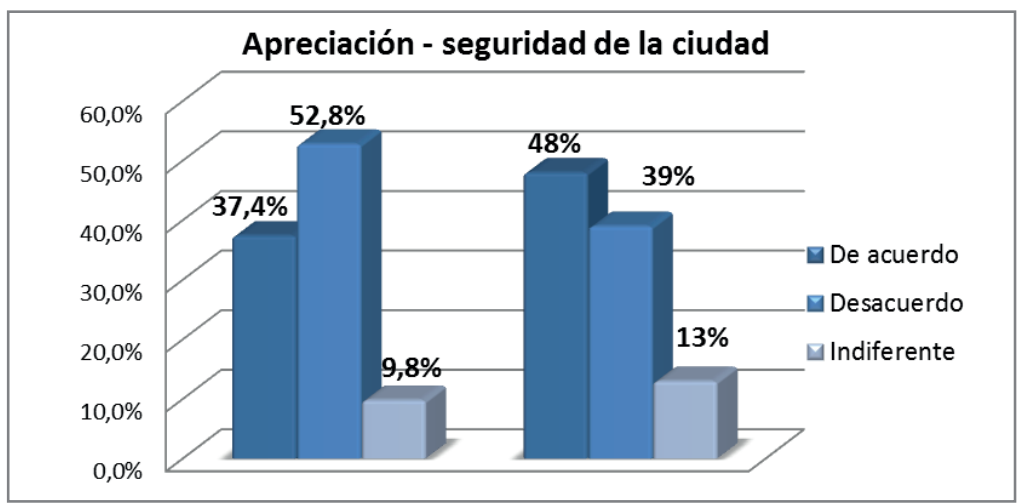

\section{Figura 4}

Apreciación de seguridad en la ciudad de Ipiales

Fuente: Elaboración propia 
City Branding como estrategia de mercado para promocionar el turismo en la ciudad de ipiales

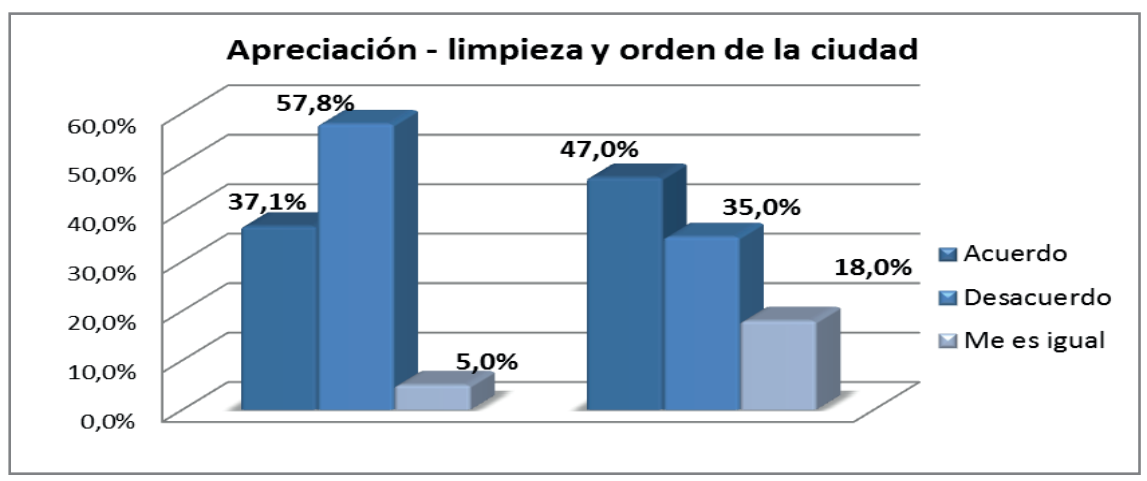

\section{Figura 5}

Apreciación de limpieza y orden de la ciudad de Ipiales

Fuente: Elaboración propia

Símbolos de la ciudad. Los residentes consideraron que los símbolos que causan mayor identidad y sentido de pertenencia están representados en el parque del 20 de julio, seguido del Santuario de las lajas y el símbolo del taita indígena. Respecto a los símbolos que tienen mayor relación con la ciudad, los visitantes consideraron el parque del 20 de julio, seguido del santuario de las lajas y el nevado Cumbal y Chiles (Ver figura 6). Estos resultados son determinantes para escoger el lugar predominante a la hora de diseñar la marca ciudad, así como buscar estrategias de promoción para estos lugares.

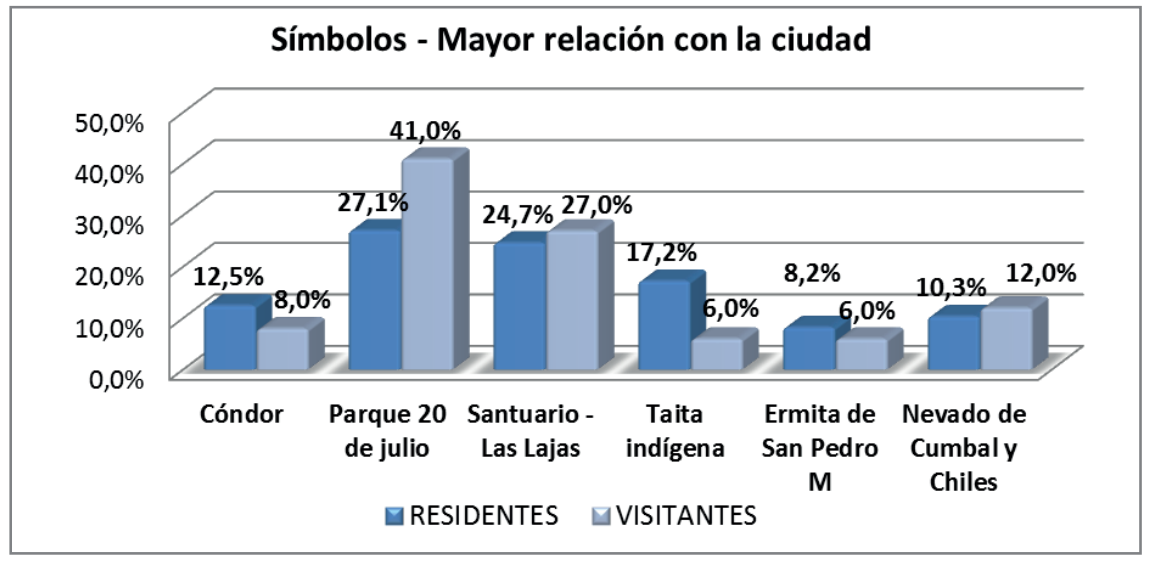

\section{Figura 6}

Símbolos de la ciudad de Ipiales

Fuente: Elaboración propia 
Colores de la ciudad. Segura, (2016), afirma que el color dentro de la vida diaria funciona como filtro de la percepción humana alterando la relación de las personas con su entorno, teniendo algunas respuestas al color que pueden ser innatas y otras que pueden ser aprendidas por la cultura. Los colores que los residentes creen que mejor representan a la ciudad, son el amarillo y el azul. Los visitantes consideraron que el color azul es el más relevante, seguido también del color amarillo y el naranja; colores representativos que serán importantes combinarlos con el color verde característico de la región. (Ver figura 7).

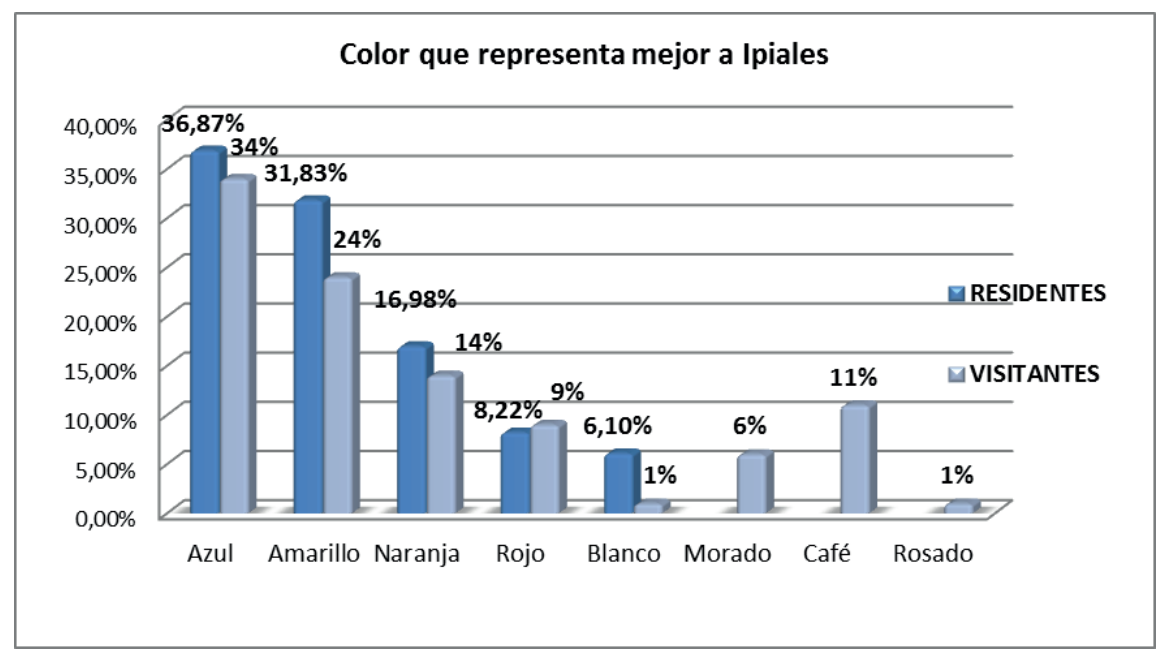

\section{Figura 7}

Colores que representa a la ciudad de Ipiales.

Fuente: Elaboración propia

\subsubsection{Otras percepciones de los residentes de la ciudad de Ipiales sobre} sentido de pertinencia y conocimiento de la marca ciudad.

Sentimiento de pertenencia hacia la ciudad. Los residentes consideraron que el ser "Ipialeño" es una de las características con las cuales se sienten más orgullosos y la que expresa el sentido de pertenencia, seguido del ser colombiano, condiciones positivas para que identifiquen y quieran la marca ciudad propuesta. (Ver tabla 1). 


\section{Tabla 1}

Apreciación del pensamiento y sentimiento del Ipialeño con respecto a su ciudad.

\begin{tabular}{|l|c|c|}
\hline Sentimiento respecto a la ciudad & Cantidad & Porcentaje \\
\hline Ipialeño & 214 & $56.8 \%$ \\
\hline Colombiano & 66 & $17.5 \%$ \\
\hline Más Ipialeño que colombiano & 47 & $12.5 \%$ \\
\hline Mas colombiano que Ipialeño & 32 & $8.5 \%$ \\
\hline Mas ecuatoriano que colombiano & 18 & $4.8 \%$ \\
\hline
\end{tabular}

Fuente: Elaboración propia.

\section{Conocimiento del concepto de marca ciudad e importancia de contar con} una marca. La mayoría de los residentes desconoce que es una marca ciudad y no ha escuchado este término. Así mismo no consideran importante contar con una marca que identifique a Ipiales. (Ver tabla 2 y 3). Estos factores no son positivos para la creación de la marca ciudad, por lo que es necesario sensibilizar a la población sobre lo importante de crear una marca, además de los beneficios para la ciudad y para sus habitantes.

\section{Tabla 2}

Conocimiento del concepto de marca ciudad

\begin{tabular}{lll}
\hline Conocimiento de marca ciudad & Cantidad & Porcentaje \\
\hline Sí & 139 & $36.9 \%$ \\
\hline No & 238 & $63.1 \%$ \\
\hline
\end{tabular}

Fuente: Elaboración propia.

Tabla 3

Importancia para los residentes de Ipiales de contar con una marca que identifique su ciudad.

\begin{tabular}{ccc}
\hline Marca & Cantidad & Porcentaje \\
\hline Me es indiferente & 278 & $73.7 \%$ \\
Me es muy importante & 99 & $26.3 \%$ \\
\hline
\end{tabular}

Fuente: Elaboración propia. 
En términos generales, los residentes de la ciudad tienen sentido de pertenencia a esta, reconocen el gran potencial turístico de Ipiales, considerando que la ciudad se favorece por sus recursos naturales, paisajes y edificaciones religiosas, lo cual lo hace apto para ser una ciudad turística, pero consideran que es indispensable mejorar sus condiciones de vías de acceso, seguridad en la ciudad y sobre todo dar a conocer y promocionar los sitios turísticos y su calidad humana.

\subsubsection{Otras percepciones de los visitantes sobre la ciudad de Ipiales.}

Estadía en la ciudad de Ipiales. Respecto al motivo principal de la visita a la ciudad e Ipiales, los visitantes consideraron principalmente el descanso o placer y visitas a familiares, seguido de compras y negocios. Resultados que son positivos para fomentar el turismo y crear una marca ciudad, (Ver tabla 4).

\section{Tabla 4}

Cuál fue el motivo principal de su visita

\begin{tabular}{lcc}
\hline Motivo principal de visita & Cantidad & Porcentaje \\
\hline Descanso o placer & 31 & $31 \%$ \\
Estudios & 10 & $10 \%$ \\
Diversión & 12 & $12 \%$ \\
Compras & 14 & $14 \%$ \\
Visita a familiares & 19 & $19 \%$ \\
Negocios & 14 & $14 \%$ \\
Cultura & 0 & $0 \%$ \\
\hline
\end{tabular}

Fuente: Elaboración propia.

Atractivos de la ciudad. Los visitantes encuestados consideraron que lo que más le llamo la atención de la ciudad es su calidez humana, seguido de los sitios turísticos y los paisajes. Resultado importante a la hora de configurar los valores y principios desarrollados al interior del municipio. 


\section{Tabla 5}

Atractivos de la ciudad

\begin{tabular}{lcc}
\hline Atractivos de la ciudad & Cantidad & Porcentaje \\
\hline Calidad Humana & 36 & $36 \%$ \\
Sitios Turísticos & 25 & $25 \%$ \\
Paisajes e Infraestructura & 18 & $18 \%$ \\
Gastronomía & 7 & $7 \%$ \\
Cultura & 5 & $5 \%$ \\
Seguridad & 2 & $2 \%$ \\
Costo de vida & 7 & $7 \%$ \\
\hline
\end{tabular}

Fuente: Elaboración propia.

Percepción de los visitantes en volver a la ciudad. Los visitantes respondieron que si volverían a la ciudad y que es de su agrado conocer más sitios junto a la cultura y tradiciones, resultados relevantes y positivos para desarrollar la propuesta de City Branding (Ver Tabla 6).

Tabla 6

Percepción de los visitantes en volver a la ciudad.

\begin{tabular}{lcc}
\hline \multicolumn{1}{c}{ Volvería a la ciudad } & Cantidad & Porcentaje \\
\hline $\mathrm{Si}$ & 95 & $95 \%$ \\
\hline no & 5 & $5 \%$ \\
\hline
\end{tabular}

Fuente: Elaboración propia.

De acuerdo a los resultados de la investigación, la ciudad presenta una serie de inconvenientes para convertirse en una ciudad turística, debido a que presenta ciertos rasgos no identitarios como problemas de seguridad, de orden público, de limpieza, demostrando el nivel cultural de la localidad y evidenciando una seria de anomalías que interfieren en que los turistas o visitantes y residentes se sientan cómodos y asuman un grado de pertenencia de identidad mayor que otras ciudades, por ello es necesario que la ciudad de Ipiales construya una identidad que produzca una impresión y recordación sensorial coordinada y asociada a su realidad, no solo para efectos comerciales e inversión, también para crear e incorporar en la comunidad propia y foránea, una identidad fuerte que, consolide una imagen exitosa. 


\subsection{Selección de la Marca Ciudad City Branding de la Ciudad De Ipiales.}

Con la caracterización general de la ciudad y los resultados de las encuestas realizadas a residentes y visitantes; se logró determinar elementos, valores culturales y humanos que pueden generar cierta identidad a lo que comúnmente se relaciona con el Ipialeño y que gracias a este tipo de información se puede estructurar modos, formas y nuevas maneras de ver a un residente y visitante de Ipiales, teniendo en cuenta componentes diversos como puede ser lo religioso, lo cultural, lo tradicional, lo verbal y lo simbólico. Por lo tanto, la importancia del Branding para Ipiales radica en posicionar la ciudad como un territorio que permita abrir las puertas a Nariño y al mundo entero y que sus ofertas turísticas sean de gran acogida y reconocimiento.

El City Branding creado para la ciudad de Ipiales, tiene como propuesta principal proyectar al público y en especial a sus turistas mediante representaciones gráficas y los elementos visuales que conforman la mixtura de Branding, como son: Marca, logotipo, símbolo, alfabeto y colores que proporcionan una expresión física para la ciudad.

Selección de la marca. Un buen nombre pude contribuir en gran medida el éxito de un producto o servicio, en este caso para el City Branding se escogió la marca "Ipiales Cultura sin Fronteras", marca que nace a raíz de una cuidadosa revisión de los resultados de las encuestas y entrevistas, no solo realizado a los turistas o visitantes sino también a los residentes de la ciudad, cumpliendo detalladamente cada una de las opiniones y sugerencias propuestas por el público objeto de estudio.

La marca "Ipiales Cultura sin Fronteras" hace un reconocimiento a Ipiales por sus connotaciones culturales, religiosas y artísticas, la cuales tienen una estrecha relación con las creencias específicamente la fe hacia la aparición de la virgen que lleva el mismo nombre de la Iglesia y que hace un reconocimiento hacia la famosa leyenda "la aparición de la virgen de las lajas", nombre que se le da al sitio donde era un camino antiguo que comunicaba a Ipiales con la frontera del país vecino del Ecuador. Así mismo, hace alusión a los procesos que conciernen los aspectos étnicos, costumbres, formas de vida que se daban en aquel entonces y que actualmente siguen siendo objeto de la vida misma de la ciudad. 
Al tomar el nombre de "Ipiales Cultura sin Fronteras", se denota y se describe los aspectos concernientes a su modo de vida, procesos y formación dentro de un entorno que hace reconocimiento a las primeras culturas o procesos de formación de la ciudad, además determina de manera fuerte la fisionomía de la ciudad junto a sus costumbres y procesos históricos. Por otro lado, se rescata los aspectos culturales y representativos de los modos de vida y los símbolos que se la conocen como la ciudad de las Nubes negras, así como también al ser zona de frontera, se resalta la tradición entorno a los lazos estrechos con el país hermano del Ecuador.

\section{Elementos fundamentales de la marca "Ipiales Cultura sin Fronteras" Den-} tro de los elementos para determinar la marca, existen características que permiten seleccionar la identidad de la misma, la primera de ellas se encuentra conformado por criterios tales como: fácil de recordar, significativo y capacidad de agradar; criterios que se constituyen en una estrategia fundamental para lograr su posicionamiento, los cuales junto al poder de adaptación, y cumplimiento de una marca que sea transferible, permiten que el Branding constituido para la ciudad de Ipiales sea el más objetable, el más significativo y el en el que convergen las distintas posiciones sobre lo que debe ser la marca ciudad para Ipiales y lo que permitiría develar los atributos físicos, religiosos, culturales y económicos en la mente no solo de los turistas sino también de los residentes.

La marca "Ipiales Cultura sin Fronteras" es fácil de recordar debido a que determina las posturas y los elementos distintivos de la ciudad en sí, ya que al decretarla como una ciudad cultural sin fronteras, saca a relucir lo más valioso y representativo de la cultura, las tradiciones de la región y sus creencias religiosas, es así como la iglesia de las Lajas se constituye como un elemento de peso en la parte turística, obra arquitectónica reconocida por su majestuosidad y por estar construida sobre un rio que artística y arquitectónicamente no solo es reconocida a nivel nacional, sino que ha dado lugar a un reconocimiento a nivel internacional ostentando el premio a una de las obras arquitectónicas consideradas una de las maravillas del mundo, de igual manera con esta marca se hace un reconocimiento a las distintas manifestaciones no solo de tipo religioso sino también de tipo artístico, gastronómico, geográfico, cultural y étnico que identifica a dicho territorio como parte importante de la historia cultural del Departamento y del País. 
Los elementos utilizados para la elaboración de la marca "Ipiales Cultura sin Fronteras" además de asumir significados, de contenido religioso, describiendo lo más relevante del territorio y a la vez categorizándolo en reducir elementos importantes del municipio, traslada lo místico y lo cultural como elementos fundamentales de la ciudad, dándoles una categoría esencial y primordial en comparación con otras, lo que permite caracterizar a la ciudad dentro de estos dos nombres o atributos y a la vez objetar lo significativo del territorio y plasmarlo a partir de una imagen o logotipo que permita fundamentar la lógica de la marca (Ver figura 8).

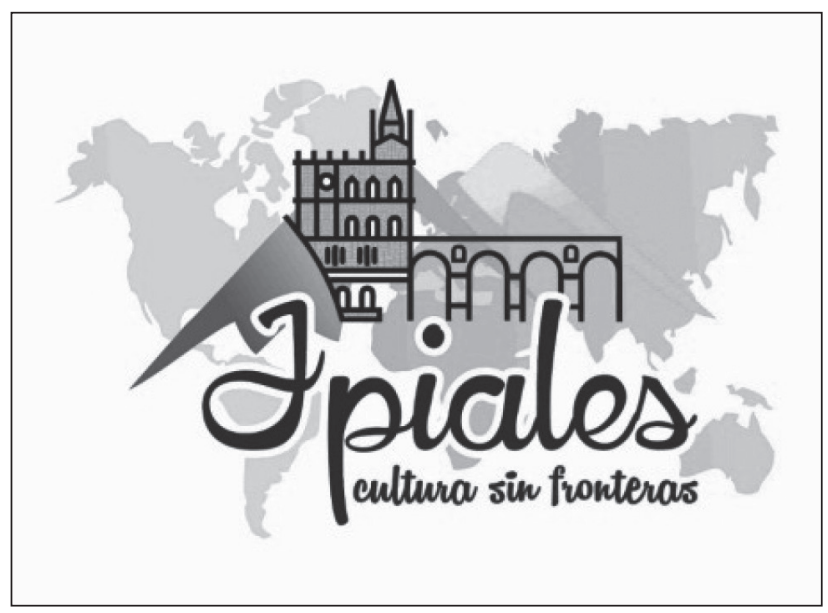

\section{Figura 8}

Diseño City Branding

Fuente: Elaboración propia

De acuerdo a American Marketing Association, determina que el logo es "un diseño gráfico que es utilizado con una continuación del símbolo por una compañía, organización o marca y a su vez se adapta a la compañía o asociación, utilizando de manera conjunta con el nombre". Para la creación y diseño del logotipo del City Branding para Ipiales se utilizó 3 colores distintivos que son la base fundamental de la ciudad, dentro de los cuales se destacan los siguientes:

Color verde: significa esperanza en un futuro mejor, el verde de los campos sembrados y el color de las nubes cuando se oculta el sol sobre las nieves del Cumbal. Color amarillo: representa ciertas connotaciones históricas, culturales y étnicas de la región. 
Color azul: es la vitalidad, la fuerza y el patriotismo de los Ipialeños.

Significado de los colores del logotipo. La marca "Ipiales Cultura sin Fronteras" refleja una imagen de la ciudad con estilo y a su vez un esquema sobre lo que representa no solo para los residentes sino también para los visitantes, ante ello, es importante considerar que los colores escogidos para el diseño del logotipo, fueron interpretados de acuerdo a las consideraciones del publico objeto de investigación, los cuales a raíz de los resultados de las encuestas consideraron que los colores verde, amarillo y azul son los de más representación y los que tienen mayor significado y relación con la ciudad.

Respecto al eslogan que es la frase que identifica a la marca, y que pretende llegar a la gente y al consumidor en este caso los turistas, el cual debe ser recordado y asociado al marketing, tendrá como objetivo permitir que "Ipiales Cultura sin Fronteras" sea renombrado y este siempre presente en la mente de los consumidores y principalmente de los visitantes y residentes.

\subsection{Estrategias de promoción del City Branding de Ipiales.}

Se propone estrategias para la promoción del City Branding, utilizando diferentes herramientas de comunicación, sensibilización y capacitación, que permitan conocer la marca ciudad no solo en la ciudad de Ipiales sino también a nivel regional y nacional. (Ver tablas 7 y 8 ).

\section{Tabla 7}

Estrategias de Promoción

\begin{tabular}{llll}
\hline \multicolumn{1}{c}{ Estrategia } & \multicolumn{1}{c}{ Descripción de la Estrategia } & \multicolumn{1}{c}{ Objetivo } & \multicolumn{1}{c}{ Medio } \\
\hline Redes & Hacer uso de las redes sociales con un & Viralizar & Virtual, \\
Sociales & comunity manager, creando perfiles en & información & aplicaciones \\
& Instagram y Facebook con contenido de la & de la marca & Instagram y \\
& marca e información turística de la ciudad. & y captar & Facebook \\
& $\begin{array}{l}\text { Promocionado las páginas y publicaciones } \\
\text { realizadas a diferentes grupos de interés. }\end{array}$ & seguidores & \\
& activos. & \\
& Trabajando con influencers y líderes de & & \\
& opinión del sector. & & \\
\hline
\end{tabular}




\begin{tabular}{|c|c|c|c|}
\hline $\begin{array}{l}\text { Puntos de } \\
\text { información } \\
\text { turística }\end{array}$ & $\begin{array}{l}\text { Ubicar puntos de información en } \\
\text { lugares estratégicos de la ciudad } \\
\text { como: Terminal terrestre, Aeropuerto, } \\
\text { Centro Comercial gran Plaza y Puente } \\
\text { internacional Rumichaca. Lugares de gran } \\
\text { afluencia de visitantes que responden } \\
\text { a temporadas turísticas y eventos } \\
\text { claramente identificados. Se ubicara un } \\
\text { stand de } 2 \times 1 \text { en los lugares indicados, } \\
\text { brindando información de la ciudad a } \\
\text { través de material P.O. (mapa turístico } \\
\text { y brochures). Que indique restaurantes } \\
\text { hoteles y tarifas de los mismos. }\end{array}$ & $\begin{array}{l}\text { Informar a } \\
\text { las personas } \\
\text { sobre los } \\
\text { diferentes } \\
\text { destinos } \\
\text { turísticos } \\
\text { de la ciudad } \\
\text { así como } \\
\text { información } \\
\text { de interés } \\
\text { para que } \\
\text { disfruten de } \\
\text { la ciudad. }\end{array}$ & $\begin{array}{l}\text { Mercaderista } \\
\text { uniformado } \\
\text { en el punto de } \\
\text { información, } \\
\text { Stand físico } \\
\text { y alianza con } \\
\text { FONTUR }\end{array}$ \\
\hline \multirow[t]{2}{*}{$\begin{array}{l}\text { Pantallas } \\
\text { y vallas } \\
\text { publicitarias }\end{array}$} & $\begin{array}{l}\text { Pautar en las pantallas publicitarias } \\
\text { ubicadas en el parque } 20 \text { de Julio, parque } \\
\text { San Felipe y Carrera } 6 \text { con calle } 17 . \\
\text { Lugares de transito obligatorio de los } \\
\text { visitantes del sur de Ipiales. }\end{array}$ & \multirow[t]{2}{*}{$\begin{array}{l}\text { Generar } \\
\text { trafico } \\
\text { visual de la } \\
\text { marca y de } \\
\text { la campaña } \\
\text { teaser }\end{array}$} & \multirow[t]{2}{*}{$\begin{array}{l}\text { Pantallas } \\
\text { digitales de } \\
\text { alto impacto } \\
\text { y vallas } \\
\text { publicitarias. }\end{array}$} \\
\hline & $\begin{array}{l}\text { Pautar en las vallas publicitarias ubicadas } \\
\text { en el Terminal de Transportes Cr 2a \# } \\
\text { 2-81 Barrio la Laguna y en las afueras del } \\
\text { centro comercial Gran Plaza. }\end{array}$ & & \\
\hline $\begin{array}{l}\text { Video City } \\
\text { Branding }\end{array}$ & $\begin{array}{l}\text { Con trasmisión en los puntos de } \\
\text { información, en las pantallas digitales y en } \\
\text { redes sociales. }\end{array}$ & $\begin{array}{l}\text { Generar } \\
\text { expectativa }\end{array}$ & $\begin{array}{l}\text { Medios } \\
\text { audiovisuales }\end{array}$ \\
\hline $\begin{array}{l}\text { Participación } \\
\text { en programa } \\
\text { radial }\end{array}$ & $\begin{array}{l}\text { Participar en el programa radial de opinión } \\
\text { Serendengue en el horario de trasmitodo } \\
\text { en la emisora RADIO VIVA } 1220 \text { AM. } \\
\text { Programa de alta frecuencia radial No. } 1 \\
\text { en la ciudad de Ipiales según la ECAR }\end{array}$ & $\begin{array}{l}\text { Dar a } \\
\text { conocer el } \\
\text { motivo del } \\
\text { cambio }\end{array}$ & $\begin{array}{l}\text { Emisora } \\
\text { Radio Viva }\end{array}$ \\
\hline
\end{tabular}

Fuente: Elaboración propia. 


\section{Tabla 8}

Actividades Alternativas de promoción

\section{Actividades Alternativas de promoción}

1. Página Web, con contenido multimedia sobre la marca, sitios turísticos e información gastronómica y hotelera de la ciudad

2. Ubicar maquetas de los sitios turísticos en el centro comercial Gran Plaza

3. Capacitar a los hoteleros y dueños de restaurantes sobre toda la información turística de la ciudad

4. Afiliar a Ipiales a la Red turística de pueblos patrimonio. FONTUR

5. Individuales y portavasos en los restaurantes más representativos con el logo de la ciudad

6. Campaña teaser con material POP en el mes de octubre en el evento Ipiales cuna de grandes tríos

7. Pantallas INDOOR Ubicadas en Aeropuerto, puente internacional Rumichaca y centro comercial Gran Plaza

8. Campaña de sensilización de la marca con entidades gubernamentales, colegios y universidades

9. Capacitación en tácticas y protocolos de atención al turista o visitante.

Fuente: Elaboración propia

\section{DISCUSIÓN}

Este escrito atiende a la necesidad de estimular la imagen e identidad de las ciudades intermedias como lo es la cuidad de Ipiales, como base para el reconocimiento urbano y posicionamiento nacional, a través de la creación de una marca que otorgue diferenciación, notoriedad y seguridad.

Ante esta temática se han realizado trabajos importantes referentes al tema de Branding como herramienta para promover una ciudad; por ejemplo existen investigaciones que buscan estrategias de marca para generar valor agregado a las actividades de un territorio o de un entorno y que a su vez genere un desarrollo social y económico impactando notablemente a la sociedad, pero consideran que a los residentes de las ciudades les falta de sentido de pertenencia; además bus- 
can promocionar las ciudades capitales como destino turístico y de inversiones y la necesidad de crear un cierto arraigo y afecto por la ciudad.

Se realizan estudios en relación a City Branding o marca- ciudad, buscando que una ciudad tome su forma, contenido y significado en la mente de las personas, permitiendo que la gente conozca y entienda la ciudad a través de sus propias percepciones creando una imagen de lo que ofrece la ciudad, por lo cual la marca ciudad deje de ser un logo o una marca turística para convertirse en un proceso de competitividad del territorio revelando atributos y características que permiten potencializar los proyectos a corto y largo plazo sobre un público potencial creando credibilidad, e intercambio de conocimientos y experiencias.

También investigaciones que ponen de manifiesto la importancia de implementar estrategias y mecanismos que permitan la introducción del City Branding para las capitales, reforzando notablemente el turismo existente y priorizando ciertos sectores económicos, sociales y culturales que hasta el momento no se han tenido en cuenta. Sumado a esto el posicionamiento estratégico online, se resalta la importancia de las páginas web como herramienta de marketing para la creación del valor de marca, es decir que las páginas web se convierten para las empresas locales de una ciudad turística en impulsoras que contribuyen a generar un City Branding.

Por lo anterior, el presente escrito no está alejado de los estudios analizados; se convierte en un punto de vista para ser viable el proyecto de City Brand en la ciudad de Ipiales, lo cual al ser una propuesta parecida permite retomar elementos teóricos y prácticos sobre lo que se entiende por marca ciudad y el manejo de una metodología apropiada para el diseño final de la propuesta, es decir, desarrollar un mecanismo que permita atribuir una función específica al proyecto de City Brand en la ciudad de Ipiales.

\section{CONCLUSIONES}

Se logró determinar una serie de elementos indispensables relacionados con el City Branding como estrategia de mercado novedosa y un mecanismo propicio para el desarrollo y posicionamiento de una marca, la cual puede contribuir a recuperar la imagen de la ciudad, siendo parte de una estrategia que determina las posibilidades para mejorar el ambiente del entorno y por consiguiente ser una 
estrategia eficaz para impulsar el turismo, además de combatir los problemas de inseguridad, de falta de oportunidades, de recreación, de diversión; es decir, que permitirá proyectar a la ciudad como un ente importante para el desarrollo turístico y una ventana para futuros proyectos de inversión.

Al contemplar la marca ciudad "Ipiales cultura sin fronteras" implica no solo tener un punto de referencia para los destinos turísticos sino que a su vez permite configurar un panorama amplio sobre las posibilidades de negocios futuros y la gestión de mercados objetivos, por lo cual se considera necesario que el planteamiento de la marca ciudad sea adaptada para el desarrollo de la región y a su vez se convierta en un punto de encuentro para la gestión de nuevas marcas y del reconocimiento de lugares estratégicos que permitan desarrollar el City Branding como estrategia mercadotécnica teniendo en cuenta sus rasgos característicos y la validez de sus conceptos teóricos y prácticos en pro del desarrollo turístico de la región.

Una vez dado el apoyo necesario por parte de las entidades públicas y privadas a este tipo de iniciativas, se considera pertinente lograr su posicionamiento dentro de las redes sociales con el fin de generar un contenido relevante para los usuarios y por consiguiente intentar que dicha marca ciudad sea reconocida no solo a nivel territorial sino también regional, nacional e internacional, logrando que el Branding para esta ciudad sea reconocido y cuente con los recursos y los dispositivos necesarios en términos tecnológicos y en manejo de redes sociales con el fin de obtener un posicionamiento que permita el uso efectivo de su marca no solo para fines turísticos sino también para finalidades empresariales, comerciales, culturales y religiosas.

Las investigaciones en este tema, son importantes desde el manejo que se dé a cada ciudad, ya que la percepción que tienen los ciudadanos puede cambiar de un sitio a otro y determinar no solo el futuro de la ciudad sino también las prácticas y vivencias que se dan al interior de los territorios, por lo cual se debe comprender que para crear una marca en una ciudad o en un territorio, se debe tener en cuenta la percepción de los habitantes acerca de los diferentes aspectos tales como seguridad, civismo, movilidad, gestión administrativa; además de aspectos relevantes entorno a la concepción sobre la vida, el medio ambiente, la economía, las posibilidades para estudiar y trabajar, percepciones que determinan en ultimas los procesos para crear un place marketing en un territorio o ciudad. 


\section{REFERENCIAS}

(1) Abadía, H. (2015). Análisis del sistema de gestión comunicacional para la construcción de una marca país-ciudad en el plan de marketing territorial de la marca Quindío de la gobernación del departamento (tesis de postgrado). Universidad Pontifica Bolivariana, Medellín, Colombia.

(2) Alcaldía municipal de Ipiales. (2016). Plan de desarrollo "Ipiales capital del SUR" 2016 - 2019. JONAS Ricardo Romero Sánchez, Alcalde Municipal. Datos Generales del municipio. [En línea] disponible en: http://Ipialescapitaldelsur.co/PLAN_DE_DESARROLLO. $\operatorname{pdf}(26 / 03 / 2018)$

(3) Bastos, L., Mogrovejo, J. y Gómez L. (2015). Experiencias del Branding como estrategia para el posicionamiento en el mercado internacional. Revista Face, facultad de ciencias económicas y empresariales, Vol. 15 No. 1, 2015 p. 3 [en línea] disponible en http://revistas.unipamplona.edu.andradeco/ojs_viceinves/index.php/FACE/article/viewFile/1812/802 $(05 / 06 / 2018)$

(4) Fernández, A. (2015). City Branding y posicionamiento online de capitales europeas en el sector turístico. España: universidad de Castilla - la Mancha, tesis doctoral, junio 2015. [En línea] disponible en https://ruidera.uclm.es/xmlui/bitstream/handle/10578/7356/ TESIS $\% 20$ Fern $\% C 3 \% A 1$ dez $\% 20 L \% C 3 \% B 3$ pez.pdf?sequence=1 (12/08/2018)

(5) Fierro, A., Zúñiga, A., Castillo, M. y Mazo C. (2015). Análisis del City Branding en la imagen de marca a través de los medios de comunicación online y la social media: caso Medellín (Colombia) revista espacios. Vol. 36 (Núm. 18) p.18 (en línea) disponible en https:// www.researchgate.net/publication/283053768_Analisis_del_City_Branding_y_la_Imagen_de_Marca_a_traves_de_los_Medios_de_Comunicacion_Online_y_el_Social_Media_Caso_Medellin_Colombia (27/07/2018)

(6) Leca, S. (2015). El Branding made in España. La marca España en la internacionalización de las marcas de calzado y moda. (tesis de postgrado). Universidad Ramón Llull, España, http://manteniment.csuc.cat/ (10/06/2018) 
(7) Mejía, L. y Bolaño, L. (2014). La calidad de las ofertas turísticas en el Departamento de la Guajira-Colombia. Revista Dimensión Empresarial, vol. 12, núm. 1, p. 139-149. . [En línea] disponible en http://www.scielo.org.co/pdf/diem/v12n1/v12n1a11.pdf(18/06/2018).

(8) Merino, M. (2014). El Branding, como herramienta para promover una ciudad. universidad nacional mayor de san marcos. (Tesis de Postgrado). Facultad de letras y ciencias humanas. Lima, Perú. [en línea] disponible en http://cybertesis.unmsm.edu.pe/bitstream/handle/ cybertesis/3762/Merino_lm.pdf?sequence=1 (2/06/2018)

(9) Muñiz, N., Cervantes, M. (2010). Marketing de ciudades y 'Place Branding'." Pecunia, 2010, p. 123. Informe Académico, (19/04/2019). . [en línea] disponible en https://go.galegroup.com/ps/anonymous?id=GALE\%7CA374628408\&sid=googleScholar\&v=2.1\&i$\mathrm{t}=\mathrm{r} \&$ linkaccess $=$ fulltext\&issn $=16999495 \& \mathrm{p}=\mathrm{IFME} \& \mathrm{sw}=\mathrm{W}$

(10) Olins, W. (2004). Las marcas según Wally Olins. Turner Publicaciones, S.L

(11) Pérez, A. y Rodríguez, I. (2014). Identidad, imagen y reputación de la empresa: integración de propuestas teóricas para una gestión exitosa. Cuadernos de gestión, Vol. 14 Num.1, Enero - Junio, 2014, pp.97-126. Universidad del País Vasco Vizcaya España.p.100 http:// www.redalyc.org/pdf/2743/274330593005.pdf (10/06/2018) (10/06/2018)

(12) Rincón, D. (2010). Urbes electrónicas: una estrategia de Citymarketing Global. Enl@ce: Revista Venezolana de Información, Tecnología y Conocimiento, 7 (1), 45-59.

(13) Rovetto, A. (2007). Hacia la construcción de una estrategia de marca ciudad para totora provincia de Santafé. (Tesis de pregrado) universidad abierta interamericana, facultad de ciencias empresariales. Rosario, Argentina. (En línea) disponible en http://imgbiblio.vaneduc.edu.ar/fulltext/files/TC077718.pdf (11/07/2018)

(14) San Clemente M., Gonzales C., y Erazo R. (2009). Formulación y evaluación de proyectos. Más que un proyecto, un plan de negocios. 2 ed. Popayán: Editorial universidad del Cauca, p 458p, ISBN: 978-958-44-4818-7

(15) Segura N. (2016). Marketing del color: ¿cómo influye el color del logotipo en la personalidad de una marca? (Tesis de Postgrado), Universidad de Chile. Santiago de Chile, Chile. http://repositorio.uchile.cl/handle/2250/140132 (25/06/2018) 